Historic, archived document

Do not assume content reflects current scientific knowledge, policies, or practices. 



\section{OAKDALE FARM}

BIRMINGHAM, ALABAMA.

Our stock of seed came from growers who are experts in the business. No better can be bought, as we do not handle the cheap and trashy kind.

We do not sell 5c packages any more, as it is not economical to either sell or buy them. We put in our 10c packages nearly three times as much seed as is put in an ordinary $5 \mathrm{c}$ package.

Our plants are grown from the very best seed we can buy. No expense is spared to secure plants that will produce big crops, and we know that is what we have. Therefore, when ordering seed and plants from us, one is assured of the very best, and at reasonable prices.

Any business sent us will receive the most careful attention.

\section{OAKDALE FARM \\ BIRMINGHAM, ALABAMA. \\ PRICES AND VARIETIES OF PLANTS}

\section{CABbage PLANTS}

Varieties: CABBAGE-Early Jersey Wakefield, Charieston Wakefield, Succession, and Flat Dutch. ONION-White Bermuda.

Prices-All varieties:

Express, not prepaid

\begin{tabular}{r|r}
300 & $\$ 50$ \\
500 & .75 \\
1,000 & 1.25 \\
5,000 & 5.00 \\
10,000 & 9.00 \\
25,900 & 2.00 \\
50.0100 & 42.50 \\
100,000 & 75.00
\end{tabular}

\begin{tabular}{c|c}
\multicolumn{2}{c}{ Postpaid Mail } \\
100 & .50 \\
200 & .70 \\
300 & .90 \\
400 & 1.10 \\
500 & 1.25 \\
600 & 1.45 \\
800 & 1.75 \\
1,000 & 2.00
\end{tabular}

\section{POTATO PLANTS}

(Ready April 1-15, depending on weather.) Varieties: Porto Rico and Nancy Hall.

Prices-All varieties:

Express, not prepaid

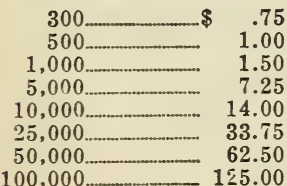

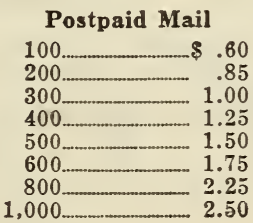

\section{TOMATO PLANTS}

(Ready April 1-15, depending on weather.) Varieties: Earliana, Livingston Globe, Greater Baltimore and New Stone.

Prices-All varieties:

Express, not prepaid
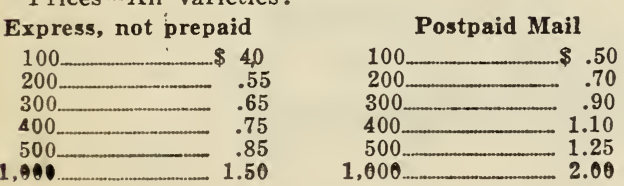

\section{PEPPER AND EGGPLANT PLANTS}

(Ready April 1-15, depending on weather.) Varieties: SWEET PEPPER - Ruoy King, Chinese Giant, Bell or Bull Nose and Pimentn. FOT PEPIPR - Long Red Cayenne. EGGPLANT-Black Beauty and New York Spineless.

Prices-All varieties:

Expicss, net prepaid

100

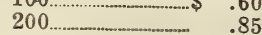

$300 \ldots-1.15$

$400 \ldots-\ldots-\ldots$

$500 \ldots-1.75$

$1,000 \ldots \ldots \ldots \ldots$

$3,000 \ldots \ldots-\ldots$

$5.000 \ldots-14.00$

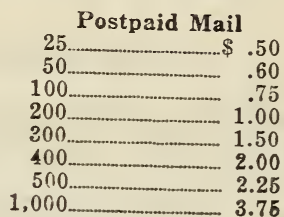

HOME GARIEN COLLECTION NO. 1

By Postpaid Mail, $\$ 1.25$

Two collections, $\$ 2.00$.

100 Tomatoes (50 early and 50 late).

25 Sweet Peppers.

10 Hot Peppers.

15 Eggplants.

\section{HOME GARDEN COLLECTION NO. 2}

By Postpaid Mail, $\$ 1.50$.

Two collections, $\$ 2.50$.

100 Tomatoes (50 early and 50 late).

50 Sweet Peppers.

25 Hot Peppers.

25 Eggplants.

\section{STRAWBERRY PLANTS}

Varieties: Klondike, Lady Thompson, Exeelsior, Aroma, and Gandy.

Priees-All varieties:

Express, not prepaid

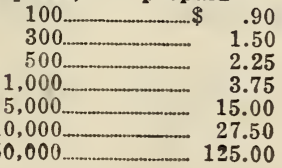

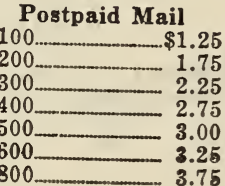




\section{CABBAGE SEED}

Varieties: Progressive and Superb.

PRICES-All varieties :

Express, not prepaid

$100 \ldots \ldots . . . . . . . . . . . . . . . . . . . . . .50$

200

1.50

300

3.25

400

500

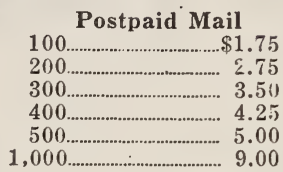

Varieties: Winter Kinc, American Drumhead Savoy, Late Flat Dutch, Late Drumhead, Autumn King, Succession, Charleston Wakefield, Early I) rumhead, Early Sumner, Early Jersey Wakefield, Early Flat Dutch, All Head Cabtage and Chinese (Pe Tsai).

Prices-All varieteis, postpaid:

$\begin{array}{cccr}\text { Pkt. } & \text { Oz. } & 1 / 4 \mathrm{tb} & 1 \text { th } \\ 10 \mathrm{c} & 25 \mathrm{c} & 75 \mathrm{c} & \$ 2.25\end{array}$

\section{PRICES AND VARIETIES OF GARDEN AND FIELD SEED}

\section{BUNCH BEAN SEED}

Varieties: Hopkins Improved Valentine, Black Valentine, Giant Stringless Green Pod, Longfellow, Refugee or 1000 to 1, Extra Early Refugee, Tennessee Green Pod, Bountiful and Improved Golden Wax.

Prices-All varieties except Stringless Green Pod and Golden Wax, not prepaid:

$\begin{array}{cccc}1 / 21 \mathrm{t} & 1 \mathrm{t} & \mathrm{Pk} . & \mathrm{Bu} . \\ 20 \mathrm{c} & 35 \mathrm{c} & \$ 3.50 & \$ 12.00\end{array}$

Giant Stringless Green Pod and Improved Golden Wax, not prepaid:

$\begin{array}{cccc}1 / 2 \mathrm{Ib} & 1 \mathrm{tb} & \mathrm{Pk} . & \mathrm{Bu} . \\ 25 \mathrm{c} & 40 \mathrm{c} & \$ 3.75 & \$ 13.00\end{array}$

\section{LIMA OR BUTTER BEAN SEED}

Varieties: Henderson's Dwarff Lima, Burpee's Bush Lima, Carolina Sieva, King of the Carden, Speckled Pole Butter Bean.

$\begin{array}{cccc}\text { Prices-All varieties, not prepaid : } & \\ 1 / 2 \mathrm{tb} & 1 \mathrm{fb} & 5 \mathrm{Hbs} & 15 \mathrm{tbs} \\ 25 \mathrm{c} & 45 \mathrm{c} & \$ 2.00 & \$ 3.75\end{array}$

\section{POLE OR RUNNING BEAN SEED}

Varieties: Striped Creaseback, White Texas Pole, Creaseback or Fat Horse, Tennessee Wonder, McCaslin Pole Bean, Texas Pole or Kentucky Wonder.

Prices-All varieties, except Texas Pole or Kentucky Wonder, not prepaid:

$\begin{array}{cccc}1 / 2 \mathrm{lb} & 1 \mathrm{tb} & \mathrm{Pk} & \mathrm{Bu} . \\ 25 \mathrm{c} & 45 \mathrm{c} & \$ 3.50 & \$ 12.50 \\ \text { Texas Pole } & \text { or Kentucky } & \text { Wonder } & \\ 1 / 2 \mathrm{tb} & 1 \mathrm{lb} & \mathrm{Pk} & \mathrm{Bu} . \\ 25 \mathrm{c} & 45 \mathrm{c} & \$ 4.00 & \$ 14.00\end{array}$

\section{COLLARD SEED}

Varieties: Georgia White Head, Georgia Blue Stem or Southern, and North Carolina. Pricos-All varieties, postpaid:

$\begin{array}{rrrr}\text { Pkt. } & \mathrm{Oz} . & 1 / 4 \mathrm{tb} & 1 \mathrm{tb} \\ 10 \mathrm{c} & 15 \mathrm{c} & 35 \mathrm{c} & 75 \mathrm{c}\end{array}$

\section{BEET SEED}

Varieties: Farly Dark Blood Turnip Beet, Extra Early Eclipse Beet, Improved Long Blood Beet. Detroit Dar'k Red Beet.

Prices-All varieties, postpaid :

$\begin{array}{cccc}\text { Pkt. } & \mathrm{Oz} . & 1 / 4 \mathrm{tb} & 1 \mathrm{tb} \\ 10 \mathrm{c} & 15 \mathrm{c} & 40 \mathrm{c} & 85 \mathrm{c}\end{array}$

\section{CAULIFLOWER SEED}

Variety: Snowball

Price, postpaid :

$\begin{array}{lll}\text { Pkt. } & \text { Oz. } & 1 / 4 \text { tb } \\ 10 \mathrm{c} & \$ 1.00 & \$ 3.50\end{array}$

\section{CELERY SEEN}

Varieties: White Plume and Golden Self Blanching.

$$
\begin{array}{ccc}
\text { Prices-All varieties, postpaid: } \\
\begin{array}{ccc}
\text { Pkt. } & 1 / 2 \mathrm{oz} . & \mathrm{nz} . \\
10 \mathrm{c} & 40 \mathrm{c} & 65 \mathrm{c}
\end{array}
\end{array}
$$

\section{SUGAR OR GARDEN CORN}

Varities: Golden Bantam, Country Gentleman, Stowe!l's Evergreen, Adams Early, and Black Mexican.

Prices-All varieties, not prepaid:

$$
\begin{array}{ccc}
1 / 2 \mathrm{tb} & 1 \mathrm{th} & 5 \mathrm{tbs} \\
15 \mathrm{c} & 25 \mathrm{c} & \$ 1.00
\end{array}
$$

\section{FIELD CORN SEED}

Varieties: Hickory King, Mammoth White Dent, Mammoth Yellow Dent. Tennessce Red Cob, Hastings Prolific. Mexica' Ju ze.

Prices-All varieties, exceyt Hastings Prolific, Mexican June, not prepaid:

$$
\text { Pk. Bu. }
$$

Prices-Hastings Prolific, Mexican June, not prepaid :

$$
\begin{array}{llll}
\text { Qt. } & \text { Pal. } & \text { Pk. } & \text { Bu. } \\
20 \mathrm{c} & 50 \mathrm{c} & 85 \mathrm{c} & \$ 2.50
\end{array}
$$

\section{CUCUMBER SEED}

Varieties: Lono Disªnce, Everrreen White Spine, Klondyke, Davis Perfect, and Long Green. Prices-All varieties. p`stpaid :

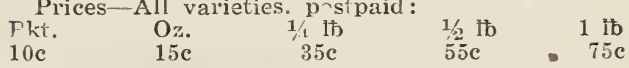

\section{CARROT SEED}

Varieties: St. Vallery, Half Long Danvers, Improved Long Orange, and Early Scarlet Short Horn.

Prices-All varieties, postpaid :

$\begin{array}{llccc}\text { Pkt. } & \text { Oz. } & 1 / 4 \text { ib } & 1 / 2 \mathrm{tb} & 1 \mathrm{ib} \\ 10 \mathrm{c} & 15 \mathrm{c} & 35 \mathrm{c} & 55 \mathrm{c} & 75 \mathrm{c}\end{array}$

\section{EGGPLANT SEED}

Varieties: New York Improved Purp!e, Florida High Bush, and Black Beauty.

Prices-All varieties, postpaid:

$\begin{array}{llllr}\text { Pkt. } & \text { Oz. } & 1 / 4 \text { tb } & 1 / 2 \mathrm{tb} & 1 \text { th } \\ 10 \mathrm{c} & 50 \mathrm{c} & \$ 1.75 & \$ 3.00 & \$ 5.50\end{array}$

\section{KALE SEED}

Varieties: Nwarf Curled Scotch, Tall Curled Scotch, and Siberian.

Prime. All varinties, postpaid:

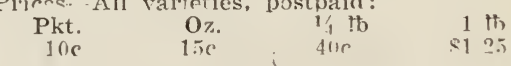




\section{OAKDALE FARM}

Birmingham, Ala.

Dear Friend:-

Herewith are our prices on fruit trees, small fruits, shrubbery, shade trees, plants, etc. These prics are as low as can be charged for high-class products, and we pride ourselves in the fact that we have no other kinc. If you want real fruit trees, plants, etc., that will give real results, send us your order.

We positively guarantee our fruit trees, plants, etc., to be true to name.

Our general price list will be found on the following pages, but we wish to call your especial attention to our TWO SPECIAL OFFERS. We refer to our offer of 100 PEACH TREES FOR $\$ 14.50$, and our HOME ORCHARD COLLECTION FOR \$6.50.

\section{HOME ORCHARD COLLECTION}

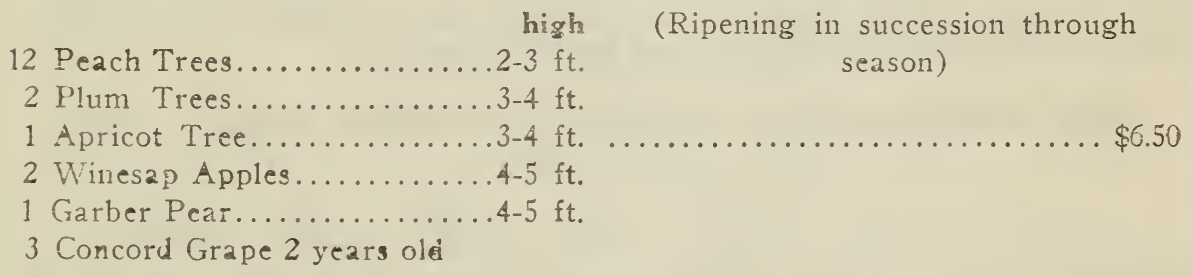

iCO PEACH TREES, 2-3 ft. high, SELECTED TO SUIT EACH SECTION, AND SO AS TO GIVE RIPE PEACHES IA SUCCESSION THROUGH SEASON, \$14.50. These 2-3 ft. Peach Trees delivered by parcel post at the following prices:

$\begin{array}{lccc}\text { Doz. } & 25 & 50 & 100 \\ \$ 4.25 & \$ 6.50 & \$ 9.75 & \$ 16.50\end{array}$

We want you to become one of our regular customers, if you are not already one. More than 5,000 folks buy from us each year, many of whom have voluntarily written us that our fruit trees, plants, etc., are the best they have ever seen. We hope to kave an order from you soon, and thank you in advance for same.

\section{Yours very truly, OAKDALE FARM.}

All prices by express, not prepaid, unless mail prices are quoted. Be sure to name express ofice.

On all orders for less than $\$ 4.00$ add 35 c for packing charges. 


\section{Price List on Fruit Trees, Small Fruits, Shade Trees, Shrubbery, Plants, Etc., From Oakdale Farm, Birmingham, Ala.}

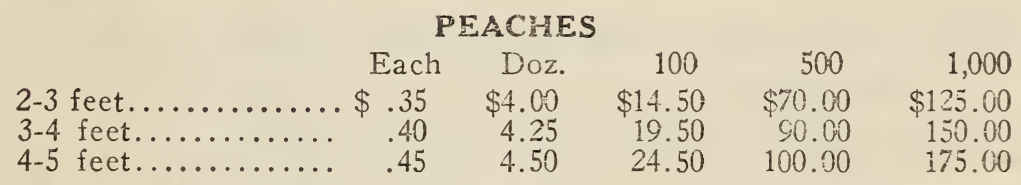

VARIETIES:-Mayflower, Victor, Yellow Swan, Greensboro, Caman, Slappey, Champion, Belle of Gecrgia, Chinese Cling, Elberta, Miftents, Greenvile Cling, Heath Cling, Salway, Wonderful, Levy, Stinson, and ỉinyeu.

\section{APPEES}

4-5 feet..........\$.50 $\begin{array}{ccccr}\text { Each } & \text { Doz. } & 100 & 500 & 1,000 \\ \$ 4.75 & \$ 29.50 & \$ 125.00 & \$ 240.00\end{array}$

VARIETIES-Early Harvest, Yellow Transparezat, Early Stravberry, Red Astrachen, Forse, Red Jure, Wilhms Eariy Red, Boman, Buchingham, Maiden Blush, Grimes, Virginia Beauty, Ben Davis, Delicious, Jonathan, Rome Beauty, Winesap, Mamnoth Black, Yates, and Rel Siberian Crab.

PEAR, PLUM, QUINCE, JAPANESE PERSIMRON, APRICOT AND MULBEREX

$$
\begin{array}{rrrrr}
\text { Each } & \text { Doz. } & 100 & 500 & 1,000 \\
4-5 \text { feet........... } \$ .7 j & \$ 7.50 & \$ 50.00 & \$ 240.00 & \$ 450.00
\end{array}
$$

VARIETIES-Pear:-Early Harvešn, Seckel, Dizhess, LeConte, Carber, and Keiffer. FLUM:-Red June, Hale, Abuadanec, Earilett, Saisina, and Dumson. JAPANESE PEESIMMON:-Tane Nasti. ADRLOT:-INonpark. MUL. BEREY:-Black English.
CHERRY

Each Doz.
100
500
1,000

$4-5$ feet........... \$.90 \$9.50 \$80.00 $\$ 350.00 \quad \$ 675.00$

VARIETIES:-Richmond, Monmorency, Governor Wood, Tartarian.

\section{PECANS}

$\begin{array}{llll}\text { Each Doz. } & 120 & 50 \% & 1.000\end{array}$

2-3 feet.......... \$1.00

$\$ 10.75$

$\$ 80.00$

$\$ 375.60$

$\$ 775.00$

$3-4$ feet.......... 1.10

11.75

$0(0.00$

425 . (it

850.00

13.75

105.00

5.00

1000.60

5-6 fec........... 1.40

15.75

12). 0 )

$5 \% 5.00 \quad 1150.00$

VARIETIES:-Schley, Stzart, Delmas, Success, And Moneymaker.

\section{FIGS}

$\begin{array}{ccccc}\text { Each } & \text { Doz. } & 109 & 500 & 1.00 \\ \$ .50 & \$ 5.00 & \$ 40.00 & \$ 175.00 & \$ 340.00\end{array}$

VARIETIES:-Brown Twhey, Brunswich, and Celestial. 


\section{BUNCH GRAPES

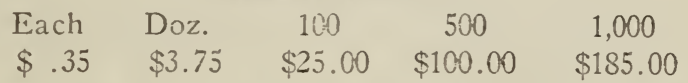

VARIETIES:-Moore's Early, Lutie, Brighton, Delaware, Niagara, Concord, and Catawaka. Add $5 c$ each vine if shipment is to be made by postpaid mail.

\section{SCUPPERNONG GRAPES}

$\begin{array}{llccc}\text { Each } & \text { D)z. } & 100 & 500 & 1,000 \\ \$ .50 & \$ 5.75 & \$ 10.00 & \$ 175.00 & \$ 325.00\end{array}$

VARIETIES:-Scuppernong, Thomas, and James. Add 5c each vine if shipment is to be made by postpaid mail.

\section{BLACKBERRY, DEWBERRY, RASPBERRY, AND ASPARAGUS

$\begin{array}{lrrr}\text { Doz. } & 100 & 550 & 1.000 \\ \$ 1.00 & \$ 3.50 & \$ 10.00 & \$ 17.50\end{array}$

VARIETIES-BLACKEERRY:-Early Harvest. DEWBERRY:-Lucretia, and Auszin. RASPBEPRY:-Cushbert, St.Regis, Cumberiand, and Columbian. ASPARAGUS:- Palmetto and Giant Argentuil. Add $20 \mathrm{c}$ per 100 if shipmen's is to be made by prepaiú mail.

\begin{tabular}{|c|c|c|c|c|c|}
\hline \multicolumn{6}{|c|}{ STRAWBERRY PLANTS } \\
\hline 100 & $30 ?$ & 500 & 1.000 & $5,0,0$ & 10,000 \\
\hline $\begin{array}{l}\text { esular Varieties.. \$ } .99 \\
\text { verbearing....... } 1.75\end{array}$ & $\begin{array}{r}\$ 1.50 \\
3.25\end{array}$ & $\begin{array}{r}\$ 2.25 \\
4.25\end{array}$ & $\begin{array}{r}\$ 3.75 \\
8.00\end{array}$ & $\$ 15.00$ & $\$ 27.50$ \\
\hline
\end{tabular}

VARIETIES-Rezular:-Klondike, Lady Thompson, Excelsior, Missionary, Aroma, and Gasidy. EVERREARING:-Progressive and Superb. Add 10c per 180 if shipment is to be made by postpaid mail.

\section{CABBAGE AND ONION PLANTS}

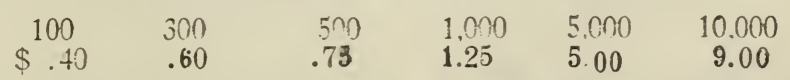

VARIETIES-CABBAGE:-Early Jersey Wakefid, Charleston Wakefield, Succession, and Flat Dutch. ONHONJ: White Bermuda. Add 5c per 109 if shipment is to be made by postpaid mail.

\section{POTATO, TOMATO, COLLARD, PEPPER AND EGGPLANT PLANTS IN SEASON}

(Write for price list when in need of any of these).

\section{SEADE TREES}

Each

Silver Maple and American White Elm....................... \$1.00

Sugar Maple, Weeping Willow and Volga Poplar........................

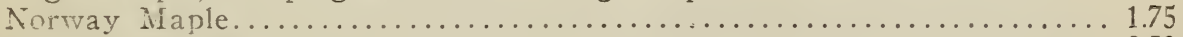

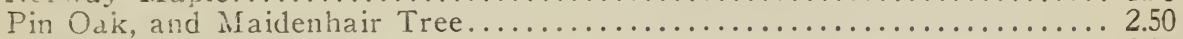

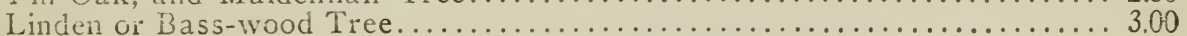

(All 6 to 9 feet high)

HEDGE PIANTS

A moor River Privet.......................................... $\$ 70$ 
AZALEA

Deutzia, Ala eagnus, Golden Bell, Althea, (Rose of Sharon), Hydrangea, Lonicera, Rosa Rugosa, Golden Elder, Spirea Van Houeti, Bridal Wreati, Purple Lilac, Snowball and Weigela.
ALL
Each
$\$ .75$

\section{BROAD-LEAVED EVERGREENS}

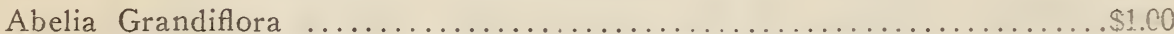

Azalea Amoena, Acuba Japonica (Green), Common Tree Box, Japanese

Holly and Magnolia Grandiflora..........................

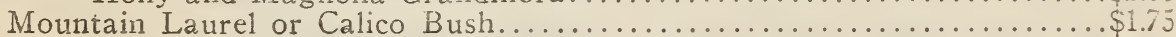

\section{CONIFEEOUS EVERGREENS}

Each

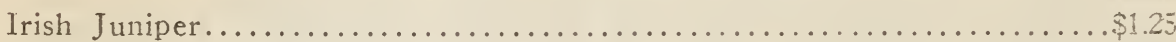

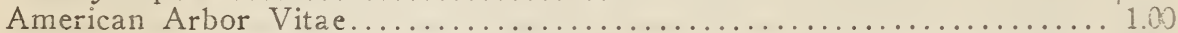

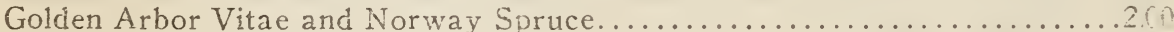

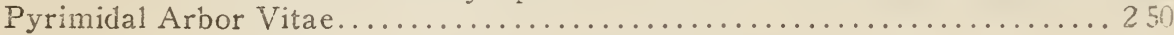

\section{VINES}

Virginia Creeper and Japanese or Boston Ivy................ \$ .75

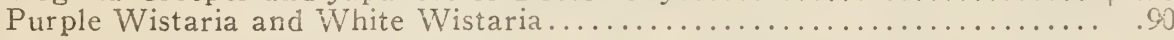

\section{ROSES}

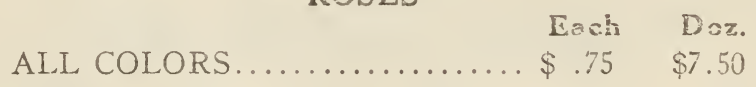

VARIETIES:- White Mamon Cochet, Pink Mamon Cocht, Kairsein, Frais

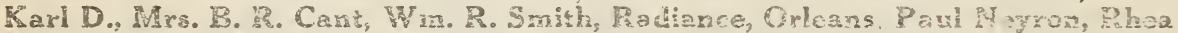

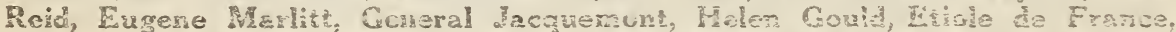
Blumenschmidt, Mme. Jems G. Mlarechal Niel, Doroty p’srkine, Exycelea, aud Gardenia.

\section{BULBS}

Hyacinths, Narcissus and Tulips, $\$ 1.25$ per dozen, not prepaid.

\section{IA AN CRASS MIXUURE}

Produces green and luxuriant grass, $35 c$ pound, not prepaid.

If you do not find in this price list what you want, write us about it, as we carry or can get any plants or nursery stock gerown.

\section{OABDALE TARM,}

Birmingham, Na. 
Varieties: Early Detroit, Earliana, Chalk's

Jewel, Livingston's Globe, Acme, Dwarf Champion, Stone, Ponderosa, Beauty and John Baer.

Prices-All varieties, except Chalk's Jewel, Acme and Ponderosa, postpaid:

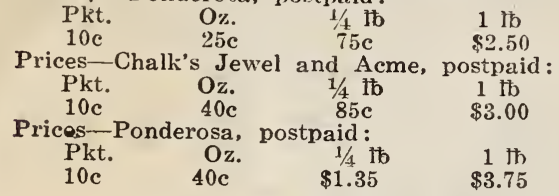

Also write us for prices when ready to buy spray pumps or any kind of spray materials. We have a complete line at reasonable prices. Spray calendar free with every order for a spray pump or spray materials. Send us your orders now.
Varieties: Sweet Alyssum, Asters, Bachelor's Button, Candytuft, Cosmos, Daisy, Four O'clock, Heliotrone, Hollyhock, Pansies, Phlox, Pinks, Petunias, Salvia, Snapdragon, Sweet William, Verbena, and Zinnia.

Prices--All varieties, postpaid:

Packet -10c.

Prices-Nasturtiums, postpaid:

\begin{tabular}{|c|c|c|c|}
\hline $\begin{array}{l}\text { Oz. } \\
15 c\end{array}$ & $\begin{array}{r}2 \mathrm{oz} . \\
25 \mathrm{c}\end{array}$ & $\begin{array}{r}1 / 4 \text { tb } \\
40 \mathrm{c}\end{array}$ & $\begin{array}{l}1 \text { tb } \\
\$ 1 \approx 5\end{array}$ \\
\hline es--Sx & Peas, & ice mixed, & postpaid: \\
\hline $\begin{array}{l}\text { Oz. } \\
20 \mathrm{c}\end{array}$ & $\begin{array}{c}1 / 415 \\
50 \mathrm{c}\end{array}$ & $\begin{array}{l}1 / 31 \mathrm{tb} \\
85 \mathrm{c}\end{array}$ & $\begin{array}{l}1 \mathrm{tb} \\
\$ 1.50\end{array}$ \\
\hline
\end{tabular}

When in the market for any kind of clovers, grass seed, peanuts, soy beans, velvet beans, cowpeas, hairy vetch, dwarf essex rapc, sorghum seed, millet, oats, wheat, rye and cotton seed, write us for prices. We have the best.

All except heavy seed like beans, corn, etc., is postpaid at prices quoted. When ordering heavy seed add postage. Your postmaster or R. F. D. man can tell you how much to send.

\section{OAKDALE FARM}

BIRMINGHAM, ALA.

\section{FREE KODAK FOR A FEW HOURS' WORK}

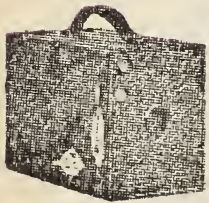

Fine Eastman Kodak given to those who will sell $\$ 10.00$ worth of our seed or plants to their friends, sending cash with order. To take advantage of this offer you must send us your order for at least $\$ 2.50$ worth of seed or plants. Your own order does not count on the $\$ 10.00$. This is merely a method of introducing our high quality seed and plants to your friends, as there is no profit to us on this proposition except in the repeat orders that we know we will get from your friends who once use our seed and plants.

This kodak makes $2 \frac{1 / 4}{4} 3 \frac{1 / 4}{4}$ pictures, and can be depended upon as being strictly first class, as that is the only kind made by the Eastman people.

Just send us your own order for seed or plants amounting to $\$ 2.50$ or more, and $\$ 10.00$ worth for one or more of your friends, and the seed $n$ plants and kodak will go forward promptly. Be sure to give the name or names of your friends to whom the seed or plants are to be shipped.

This is an opportunity to easily earn a good kodak, as all of your friends will buy seed or plants. Start to work now.

\section{OAKDALE FARM \\ BIRMINGHAM, ALA.}

